\title{
Corela
}

Cognition, représentation, langage

HS-8 | 2010

L'interpellation

\section{Norme, idéologie, imaginaire. Les rituels de l'interpellation dans la perspective d'une philosophie du discours}

\section{Marie-Anne Paveau}

\section{OpenEdition}

Journals

Édition électronique

URL : http://journals.openedition.org/corela/1797

DOI : 10.4000/corela. 1797

ISSN : 1638-573X

Éditeur

Cercle linguistique du Centre et de l'Ouest - CerLICO

Référence électronique

Marie-Anne Paveau, « Norme, idéologie, imaginaire. Les rituels de l'interpellation dans la perspective d'une philosophie du discours », Corela [En ligne], HS-8 | 2010, mis en ligne le 23 novembre 2010, consulté le 20 avril 2019. URL : http://journals.openedition.org/corela/1797 ; DOI : 10.4000/ corela. 1797

Ce document a été généré automatiquement le 20 avril 2019.

\section{(c) (1)(2)}

Corela - cognition, représentation, langage est mis à disposition selon les termes de la licence Creative Commons Attribution - Pas d'Utilisation Commerciale - Partage dans les Mêmes Conditions 4.0 International. 


\title{
Norme, idéologie, imaginaire. Les rituels de l'interpellation dans la perspective d'une philosophie du discours
}

\author{
Marie-Anne Paveau
}

\section{Introduction}

1 Je souhaiterais envisager l'interpellation dans ses dimensions historique, sociale et politique, comme un rituel de la vie humaine. Sous cet angle, l'interpellation peut être vue comme une forme d'intersubjectivité, le terme désignant précisément, à partir de la tradition husserlienne, l'existence d'une pluralité de sujets qui communiquent entre eux et partagent un monde commun présent à la conscience de chacun :

[...] on peut considérer d'ores et déjà comme établi, le fait que j'ai en moi, dans le cadre de ma vie de conscience pure transcendentalement réduite, l'expérience du « monde » et des " autres " - et ceci conformément même au sens même de cette expérience, - non pas comme d'une œuvre de mon activité synthétique en quelque sorte privée, mais comme d'un monde étranger à moi, « intersubjectif », existant pour chacun, accessible à chacun dans ses objets (Husserl 1992 [1947] : 150). La notion est retravaillée par le structuralisme dans le cadre de l'affaiblissement du sujet (c'est l'origine d'intertextualité par exemple), saisi par la psychanalyse lacanienne, par la linguistique de l'énonciation (Émile Benveniste emploie le terme dans son article de 1958, «La subjectivité dans le langage » pour soutenir la notion de discours), par la sémantique référentielle (Georges Kleiber parle de «stabilité intersubjective» du sens) et par certaines approches cognitives non orthodoxes (c'est-à-dire non internalistes, j'y reviendrai).

3 Mon propos est donc, dans la perspective de ce que j'appelle une philosophie du discours, qui implique nécessairement une histoire et même une éthique du discours, d'inscrire l'interpellation dans le cadre de l'intersubjectivité et de rendre compte de son fonctionnement, comme forme intersubjective nécessaire, dans trois domaines 
disciplinaires privilégiés, qui sont des lieux d'articulation entre la linguistique et ses disciplines affines ou contributives. Il s'agit de: l'histoire sociale du langage (en fait la Social history of language britannique, champ qui n'existe pas véritablement en France comme tel mais qui correspondrait à quelque chose comme une sociolinguistique ou une sociopragmatique historique), la théorie du discours marxo-freudienne (projet qui articule la linguistique, la philosophie et la psychanalyse entre 1960 et 1985) et, de l'autre côté de l'Atlantique, la gender theory, au croisement de la philosophie, de la rhétorique et de la pragmatique, qui développe une théorie de la performativité.

4 La référence commune et englobante est l'approche philosophique, qui fait une place centrale à la catégorie de l'interpellation, qui est au départ une catégorie discursive de philosophe et qui peut, moyennant des aménagements théoriques et épistémologiques, devenir une catégorie linguistique de linguiste.

\section{1. «On ne dit pas M'sieurs-dames »: les normes socio-historiques de l'interpellation}

5 L'interpellation comme rituel social, donc constituant un contact ou manifestant une relation sociale, aussi éphémère soit-elle, est une forme d'intersubjectivité nécessaire à la vie sociale. Comme rituel social, l'interpellation est soumise à des paramètres historiques et culturels.

\subsection{Pour une histoire sociale du langage/discours}

On n'interpelle pas n'importe qui n'importe comment n'importe où selon les époques, les cultures et les rapports sociaux, comme le montre Liselotte (auteure d'un célébrissime guide des convenances dans les années 1920) dans la première exergue à cet article, ce qui nécessite l'appel à des données socio-historiques. C'est ce que fait par exemple la Social history of language, développée en Grande-Bretagne, en particulier à travers les travaux de et initiés par Peter Burke sur la dimension linguistique de l'histoire sociale. Dans le premier recueil qu'il dirige avec Roy Porter en 1987, sont par exemple envisagés les formes de politesse et d'insulte ou les formes langagières de la révérence et de la révolte. Dans le second recueil de 1991, les objets évoluent un peu vers les formes langagières du pouvoir (y compris dans la dimension interlinguistique, c'est-à-dire sur le latin ou l'hébreu par exemple comme langues de l'autorité), le pouvoir symbolique constitué par certains usages langagiers (dans une perspective très proche de celle de Pierre Bourdieu). En France, ce qui pourrait s'appeler une « histoire sociale des normes langagières ou des mœurs langagières » est prise en charge essentiellement par :

7 - la littérature, dans un secteur circonscrit, celui des travaux d'Alain Montandon et de son équipe de Clermont-Ferrand à l'œuvre dans le Dictionnaire de la politesse et du savoir-vivre qu'il a dirigé en 1995 ;

- d'autres disciplines comme l'histoire, avec de rares travaux comme ceux d'Éric Mension-Rigau sur les classes dominantes par exemple, qui prennent en compte la dimension sociolectale (Mension-Rigau 1997);

- le champ de la sociolinguistique, bien sûr, mais qui n'intègre pas ou très peu la dimension historique: depuis quelques années se développe le champ relativement nouveau mais non fédéré de la « violence verbale " (pour une synthèse, voir Moïse et al. 
2008 par exemple), auquel on ajoutera des chercheuses comme Dominique Lagorgette et Laurence Rosier, ainsi que le groupe de politistes de l'université de Dijon qui possède un site internet consacré à l'insulte en politique (http://passerelle.u-bourgogne.fr/ publications/atip_insulte). Ce champ pourrait relever de la Social history of language à la française, avec une dominante plus sociologique ou politique qu'historique cependant. Je remarque cependant que les objets étudiés sont toujours des formes transgressives, des indices de rupture de l'ordre social, et que l'ordre social lui-même accompagné de ses normes « positives » est rarement construit en objet pour la linguistique concentrée sur le seul ordre de la langue. - l'interactionnisme, dans une très petite proportion cependant: les travaux de Catherine Kerbrat-Orecchioni par exemple s'appuient beaucoup sur ceux des Anglo-Saxons et prennent assez peu en compte les dimensions historique et sociologique ;

- mais surtout, dans une très grande proportion, un immense corpus de linguistique populaire ou profane ${ }^{1}$, plus précisément une sociolinguistique populaire ou sociolinguistique spontanée dont Pierre Bourdieu réclamait d'ailleurs la «science rigoureuse » dès 1983 dans son article « Vous avez dit populaire? » (dans Bourdieu 2001 : 137), sans avoir été vraiment entendu ces vingt dernières années. Le corpus de linguistique profane est constitué de manuels de savoir-vivre, de guides de correspondance, d'une littérature que l'on peut appeler «l'esprit français » (Pierre Daninos, Robert Beauvais, Philippe Jullian), et qui fait l'objet de travaux émergents en France actuellement (Achard-Bayle, Paveau 2008, Achard-Bayle, Lecolle 2009 (à par.), Paveau 2007, 2008, Paveau, Rosier 2008).

8 À la question: comment aborder la dimension linguistique de l'interpellation comme rituel social ?, je propose une réponse qui puise ses informations dans les données de la sociolinguistique populaire.

\subsection{L'interpellation : un rituel social explicite}

9 L'interpellation, on l'a vu, n'est pas une catégorie linguistique stabilisée mais elle se laisse décrire cependant (voir la contribution de Catherine Détrie ici même) et englobe les termes d'adresse. On trouve en sciences du langage un certain nombre de travaux sur les termes d'adresse dans une perspective formelle, pragmatique ou microcontextuelle, mais assez peu de réflexions sur leur pertinence sociale et relationnelle dans les groupes, qu'ils soient professionnels, sociaux ou générationnels. La linguistique, même sociale, se dit peut-être, comme la philosophie, qu'elle n'a pas de dialogue à avoir avec ce qu'Alain Montandon appelle « la morale sociale collective» :

En effet la politesse n'est qu'un chapitre mineur de la morale sociale collective, « la plus humble section de la philosophie » pour Erasme. Elle ne concerne véritablement ni la morale, car un manquement aux convenances est sans doute une faute de goût mais non une dérogation à l'éthique, ni le droit, car une impolitesse n'est pas un délit et ne relève d'aucune juridiction. La transgression n'encourt que la blâme d'autrui, un regard de reproche, un jugement de désapprobation. La politesse relève de la coutume, des usages et des habitudes sociales, de ce qu'on appelle les « devoirs mineurs» (Montandon (dir.) $1995: 5$; préface).

11 Il existe quelques exceptions à cette mise à distance, par exemple les travaux de Felicia Dumas sur les titres religieux en milieu orthodoxe (Felicia Dumas 2008), dans lesquels sont examinés les différentes normes d'emploi des formes et formules d'adresse selon le 
code de savoir-vivre en vigueur dans cette communauté. C'est dans cette perspective que je choisis ici l'exemple des termes d'adresse dans les formules de salut en français dans la société française, à travers un corpus de manuels de savoir-vivre entre 1952 et 2004 (comprenant un ouvrage humoristique de Jean-Louis Fournier riche en informations sociodiscursives) :

\section{Manuels de savoir-vivre 1952-2004}

- Quercize F. (de), 1952, Guide des bons usages dans la vie moderne, Paris, Larousse. 99 : Ne dites pas : Bonjour, Messieurs-Dames - Dites : Bonjour, Monsieur ; Bonjour, Madame 154 : On ne dit jamais « Bonjour, Monsieur Dupont » ou « Comment allez-vous, madame Lenoir ", cela s'emploie à l'égard des subordonnés.

13 - Forel D., 1962, Le vrai savoir-vivre. Heure par heure en toutes circonstances, Marabout flash, Verviers, Gérard et $\mathrm{C}^{\circ} .49$ : Le nom des gens : sauf au moment des présentations, il ne se prononce jamais.

14 - Weil S., 1983, Trésors de la politesse française, Paris, Belin. 11: «Bonjour messieursdames!». Cette formule, honnie de tous temps par les puristes qui la disent incorrecte et par les gens « chics » qui la trouvent vulgaire, s'emploie pourtant beaucoup, notamment dans les grands magasins. 12: Dans les relations mondaines, on évitera le «Bonjour, Madame (ou Monsieur) Durand» considéré comme particulièrement cordial à la campagne, au marché, dans le commerce et, surtout au masculin, dans les relations professionnelles.

15 - Guibert M., Delamarque P., 1990, Savoir recevoir savoir vivre, Paris, France Loisirs. 58 : N'omettez jamais le mot « Madame » quand vous vous adressez à une dame, «Monsieur » quand il s'agit d'un homme, ni même le prénom des gens que vous traitez plus familièrement. Ne dites par « Madame Martin », etc.

16 - Fournier J.-L., 1998, Je vais t'apprendre la politesse, Paris, Le livre de poche. Bonjour, monsieur. Bonjour, madame. Dans les relations commerciales, n'hésitez pas à montrer à la personne que vous vous rappelez son nom, dites : «Bonjour monsieur Dupont » Mais seulement à monsieur Dupont.

17 - Cechman O., 2004, Le guide du nouveau savoir vivre. Le bon usage, Paris, Hachette. Avant tout, dire " bonjour », « pardon », « s'il vous plaît », « au revoir ». En faisant suivre selon les cas de «monsieur » ou « madame » pour toutes les personnes que vous vouvoyez. Quelle analyse socio-historique peut-on faire des appellatifs à partir de ces remarques ?

On remarque immédiatement deux traits saillants: les remarques sont principalement des proscriptions ("ne dites pas», "on ne dit jamais») ou des restrictions («mais seulement à...»), et une évolution nette se dessine entre 1952, dans le sens d'une simplification des remarques et d'une relativisation des consignes («selon les cas »). On pense évidemment aux analyses de Norman Fairclough dans Discourse and social change en 1992, qui souligne la "démocratisation » et la "conversationnalisation » du discours et des façons de parler, à propos de l'anglais, les analyses étant partiellement exportable aux pays européens dont la France. Norman Fairclough appelle démocratisation un phénomène plus large que l'adoucissement des hiérarchies par les termes d'adresse, puisque cela concerne aussi les "prises de pouvoir » conversationnelles. Il en donne la définition suivante 1. Democratization of discourse By "democratization" of discourse I mean the removal of inequalities and asymmetries in the discursive and linguistic rights, 
obligations and prestige of groups of people. [...] elimination of overt power markers in institutional discourse types with unequal powerrelations. [...] asymmetrical terms of address ; 'bald' (eg imperative) directives, in favour of more indirect and 'face'-sensitive forms (Brown and Levinson 1978); asymmetries in rights to make certain sorts of contribution, such as initiating topics, and asking questions; use by powerful participants of specialized vocabulary inacessible to others (Fairclough $1992: 200$ et ss.).

Catherine Kerbrat-Orecchioni résume ce point de vue en parlant de «l'affaiblissement de la compétence appellative de la communauté parlante » $(1992$, p. 54). On pourrait faire le même type d'analyse sur les titres, avec des îlots de conservation comme l'armée par exemple, et des comparaisons interculturelles (en Italie ou en Estonie, par exemple, et dans d'autres cultures encore, l'appellatif par titre est encore très vivace tant à l'université qu'en d'autres lieux apparemment moins formels comme... la pharmacie).

21 Mais ce qui est intéressant ici à propos du phénomène sociodiscursif des appellatifs, c'est le parallèle étroit qui existe entre linguistique savante et linguistique profane, puisqu'en 1999 par exemple, Maurice Druon, l'un des plus éminents linguistes sauvages de France, propose le même type d'analyse, sous une forme évidemment très différente. Il s'agit d'une explication d'un phénomène pragmatico-lexical, l'emploi de tout à fait et absolument comme réponses à une question : «C'est sans doute parce qu'on s'est défait d'une certaine habitude de politesse qui voulait qu'on dise: "oui, monsieur, oui, madame ; oui, mon père ; oui, mon oncle » (Maurice Druon, Le bon français, 1999: 50). Sur la question des appellatifs et des normes de politesse discursive en général, le corpus profane est une source de première main, tant sur le plan des informations que sur celui des analyses. Pas plus que le corpus savant il ne répond cependant à la question de la nature exacte des évolutions sociales apparemment impliquées par la démocratisation appellative. «Les modes de coordination sociale », selon l'expression de Françoise Gadet, constituent en effet une évolution de la relation sociale elle-même, phénomène qui possède une dimension philosophique, du côté politique et éthique. La question est de savoir à quel niveau se situent les évolutions historiques, à un niveau seulement cosmétique qui conserve les rapports de pouvoir et leur violence, ou un niveau profond qui adoucit véritablement les mœurs.

22 Je vais essayer de répondre à cette question en faisant un détour par l'esquisse d'une histoire conceptuelle de l'interpellation ente la France et les États-Unis.

\section{2. « Oui, c'est bien moi ! » : idéologie et construction discursive du sujet}

23 L'interpellation, dans la perspective d'une philosophie du discours, s'inscrit dans un ensemble d'autres concepts qui lui sont articulés: attachement, assujettissement, assignation, affiliation, reconnaissance, justification, qui relèvent d'une manière de voir les relations sociales de pouvoir à partir d'un choix théorique et/ou politique. Je privilégie ici ceux qui sont directement concernés par la dimension discursive, et je voudrais retracer brièvement les migrations d'un concept, celui d'interpellation, qui passe de la philosophie (le matérialisme dialectique) à l'analyse du discours (la sémantique discursive de Michel Pêcheux) en ayant fait un crochet par la psychanalyse, et qui émigre aux États-Unis où il est intégré à la French Theory qui nous revient 
actuellement sous les formes de la théorie de la performativité au centre de la gender theory.

\subsection{Idéologie, inconscient : l'interpellation chez Althusser}

Je privilégie ici les propositions d'Althusser sur l'interpellation plutôt que celles de Foucault sur l'assujettissement pour des raisons à la fois lexicales, disciplinaires et conceptuelles : Althusser emploie le terme d'interpellation et propose une véritable scène discursive de l'interpellation, alors que Foucault, moins langagier que discursif (et encore son emploi de la notion de discours est-il assez éloigné, comme on le sait, du discours des linguistes), préfère le terme d'assujettissement sans en donner une illustration qui concerne directement le langage.

Althusser écrit en 1969 le célèbre article intitulé «Idéologie et appareils idéologiques d'État. Notes pour une recherche ", qui sera publié en juin 1970 dans La pensée puis repris dans Positions et le recueil Sur la reproduction édité par Actuel Marx en 1995; dans cet article désormais classique, on trouve le non moins classique passage où la notion d'interpellation est précisément définie :

Comme le disait admirablement Saint Paul, c'est dans le «Logos », entendons dans l'idéologie, que nous avons « l'être, le mouvement et la vie». Il s'ensuit que, pour vous comme pour moi, la catégorie de sujet est une «évidence » première (les évidences sont toujours premières) : il est clair que vous et moi sommes des sujets (libres, moraux, etc.). Comme toutes les évidences, y compris celle qu'un mot "désigne une chose» ou "possède une signification» (donc y compris les évidences de la «transparence» du langage), cette «évidence » que vous et moi sommes des sujets - et que ça ne fait pas problème - est un effet idéologique, l'effet idéologique élémentaire. C'est en effet le propre de l'idéologique que d'imposer (sans en avoir l'air, puisque ce sont des "évidences»), les évidences comme évidences, que nous ne pouvons pas ne pas reconnaître, et devant lesquelles nous avons l'inévitable et naturelle réaction de nous exclamer (à haute voix, ou dans le « silence de la conscience ») : « C'est évident ! C'est bien ça ! C'est bien vrai ! ».

Dans cette réaction s'exerce la fonction de reconnaissance idéologique qui est une des deux fonctions de l'idéologie comme telle (son envers étant la fonction de méconnaissance) - (Althusser $1970: 30$ ).

C'est à partir des notions d'évidence et de reconnaissance que s'élabore celle d'interpellation, elle-même constituant centralement le sujet, comme on le voit une page plus loin :

Je dirais dans une première formule: toute idéologie interpelle les individus concrets en sujets concrets, par le fonctionnement de la catégorie de sujet. [...] Nous suggérons alors que l'idéologie "agit » ou "fonctionne» de telle sorte qu'elle « recrute » des sujets parmi les individus (elle les recrute tous), ou "transforme » les individus en sujets (elle les transforme tous) par cette opération très précise que nous appelons l'interpellation, qu'on peut se représenter sur le type même de la plus banale interpellation policière (ou non) de tous les jours : « hé, vous, là-bas! ». [...] L'expérience montre que les télécommunications pratiques de l'interpellation sont telles, que l'interpellation ne rate quasiment jamais son homme : appel verbal, ou coup de sifflet, l'interpellé reconnaît toujours que c'était bien lui qu'on interpellait (Althusser 1970 : 31)

Comprenons bien ce qu'Althusser veut dire : il n'y a pas d'autonomie de la catégorie de sujet, elle ne peut exister que par l'interpellation. Cette dernière lui préexiste donc, et non l'inverse. Comme le montre bien l'incipit «Comme le disait admirablement Saint Paul », cette proposition sur l'interpellation de l'individu en sujet est faite à partir d'une 
réflexion sur le discours religieux. C'est explicité un peu plus loin, où se trouve la formule apparemment innocente de "Oui c'est bien moi » qui me sert de sous-titre et qui est en fait lourdement chargée de ce que la religion peut avoir d'autocratique et de clôturé pour Althusser :

L'idéologie religieuse chrétienne dit à peu près ceci. Elle dit : Je m'adresse à toi, individu humain appelé Pierre (tout individu est appelé par son nom, au sens passif, ce n'est jamais lui qui se donne son Nom), pour te dire que Dieu existe et que tu lui dois des comptes. [...] Or c'est là un discours tout à fait connu et banal, mais en même temps tout à fait surprenant... Surprenant, car si nous considérons que l'idéologie religieuse s'adresse bien aux individus pour les 'transformer en sujets', en interpellant l'individu Pierre pour en faire un sujet, libre d'obéir ou de désobéir à l'appel, c'est-à-dire aux ordres de Dieu ; si elle les appelle par leur Nom, reconnaissant ainsi qu'ils sont toujours - déjà interpellés en sujets ayant une identité personnelle [...]; si elle les interpelle de telle sorte que le sujet répond 'oui, c'est bien moi !'; si elle obtient d'eux la reconnaissance qu'ils occupent bien la place qu'elle leur désigne comme la leur dans le monde, une résidence fixe : 'c'est bien vrai, je suis ici, ouvrier, patron, soldat!' dans cette vallée de larmes; [...] si tout cela se passe bien ainsi (dans les pratiques des rituels bien connus...), nous devons remarquer que toute cette 'procédure', mettant en scène des sujets religieux chrétiens, est dominée par un phénomène étrange: c'est qu'il n'existe une telle multitude de sujets religieux possibles que sous la condition absolue qu'il y ait un Autre Sujet Unique, Absolu, à savoir Dieu... (Althusser 1970 : 116-118).

En d'autres termes, le statut de sujet constitue l'illusion suprême fabriquée par l'idéologie religieuse; sur ce modèle, l'interpellation est au cœur d'une théorie discursive du pouvoir. L'on va voir que ce pouvoir, à l'origine de la théorisation, est celui de l'inconscient, l'un des grand déterminismes présents dans la théorie du discours des années structuralistes.

\subsection{Les origines freudo-lacaniennes de l'interpellation idéologique}

Si l'on replace cet article dans l'ensemble du corpus althussérien, et si on le met en regard des Écrits sur la psychanalyse, on constate que quasiment toute la théorie de l'interpellation idéologique est présente dès 1966 dans les lettres à Diatkine. Il y a en effet un lien très étroit entre inconscient et idéologie, fait un peu oublié par les discursivistes actuels qui remettent au travail la notion d'idéologie, cet oubli lui enlevant à mon avis une grande partie de son efficace. C'est le sens de ma seconde exergue qui met en scène le fou, la poule et le grain de blé.

Althusser utilise la métaphore du moteur pour définir, dans le cadre de la psychanalyse, en 1966, donc quatre ans avant l'article de La pensée, ce qu'il appelle idéologie :

Nous sommes d'accord qu'une fois constitué, l'inconscient fonctionne comme une structure « intemporelle ». J'userai ici d'une comparaison: une fois qu'il a été monté, et monté pour être capable de fonctionner, un moteur "fonctionne" toujours à quelque chose. Par exemple un moteur à essence fonctionne à l'essence. Or je me demande si on ne peut pas dire que l'inconscient a lui aussi besoin de "quelque chose" pour fonctionner: et ce "quelque chose» est, me semble-t-il, en dernier ressort de l'idéologique. Sur ce point, il faudrait que j'esquisse une explication de ce qu'est l'idéologique. Il suffit pour notre propos présent de savoir que l'idéologique ne se réduit pas aux systèmes conceptuels de l'idéologie, mais est une structure imaginaire qui existe 
non seulement sous la forme de concepts, mais aussi sous la forme d'attitudes, de gestes, de conduites, d'intentions, d'aspirations, de refus, de permissions, d'interdits, etc. (Althusser 1966: 108; «Lettres à D...»). Quand Althusser emploie le mot d'imaginaire, c'est évidemment, dans le contexte des Lettres à son analyste et dans celui, plus large, de la pensée des années 1960, à l'imaginaire lacanien qu'il pense. Dans la pensée de Lacan, l'imaginaire, au sein du système RSI (Réel, Symbolique, Imaginaire), est une « représentation leurrante »:

Dans la méconnaissance, le refus, le barrage opposé à la réalité par le névrotique, nous constatons un recours à la fantaisie. Il y a la fonction, ce qui dans le vocabulaire de Freud ne peut renvoyer qu'au registre imaginaire. Nous savons combien les personnes et les choses de l'entourage du névrosé changent entièrement de valeur, et ce par rapport a une fonction que rien ne fait obstacle à désigner - sans chercher au-delà de l'usage commun $\mathrm{du}$ langage - comme imaginaire. Imaginaire renvoie ici - premièrement, au rapport du sujet avec ses identifications formatrices, c'est le sens plein du terme d'image en analyse deuxièmement, au rapport du sujet au réel dont la caractéristique est d'être illusoire, c'est la face de la fonction imaginaire le plus souvent mise en valeur (Lacan 1975 [1953-1954], Séminaire $1: 63$ 186).

33 À partir des élaborations ultérieures, on peut définir l'imaginaire comme un ensemble de représentations inconscientes, dont l'existence est liée au clivage du sujet. Dans le moi il engendre la fonction de méconnaissance (de l'Autre sur l'Un); dans la relation intersubjective il engendre la projection (de l'Un sur l'Autre). L'imaginaire implique bien sûr la dénégation. L'idéologie comme structure imaginaire, c'est donc un pouvoir assujettissant dont le sujet n'a pas conscience mais auquel il se soumet tout en le déniant : «Mais oui, c'est moi », dit-il en se retournant et en affirmant sa liberté de se retourner. C'est ainsi que Lacan peut définir le sujet comme victime de son propre leurre :

Cette conviction dépasse la naïveté individuelle du sujet qui croit en soi, qui croit qu'il est lui - folie assez commune, et qui n'est pas une complète folie, car cela fait partie de l'ordre des croyances. Évidemment, nous avons tous tendance à croire que nous sommes nous. Mais nous n'en sommes pas si sûrs que ça, regardez-y de bien près (Lacan 1978 [1954-1955] Séminaire $2: 20$ ).

35 C'est en ce sens que le sujet est un "effet-sujet ", un effet de l'interpellation idéologique, un effet de l'imaginaire. La notion d'effet, elle aussi largement effacée dans les analyses du discours contemporaines, est pourtant centrale dans les élaborations théoriques les plus robustes de cette discipline.

\subsection{L'effet comme produit discursif de l'interpellation idéologique : la théorie du discours}

C'est par la notion d'effet, entre autres, que la théorie matérialiste de l'idéologie vient nourrir la théorie du discours de Michel Pêcheux et de son groupe, à partir de la fin des années 1960. Une des autres notions centrales dans l'analyse du discours dite " française », le préconstruit, proposé par Paul Henry, est elle aussi directement liée au marxisme et à la psychanalyse, et articulée à la notion d'effet-sujet, comme on va le voir. On trouve une des premières formulations de l'effet-sujet dans un travail inédit de Catherine Fuchs et Michel Pêcheux, qui porte sur les trois mécanismes permettant de différencier le « conceptuel-scientifique » et le «notionnel-idéologique »: 

une des propositions théoriques les plus fortes d'une analyse du discours qui se saisit du «réel de la langue », c'est-à-dire de l'Autre, la part insue de la langue et du sujet. Je renvoie à l'introduction de L'inquiétude du discours par Denise Maldidier, au «Que sais-je ?» de Francine Mazière sur l'analyse du discours et je me permets de renvoyer à ma propre synthèse sur la notion de préconstruit et les concepts liés qui concernent les connaissances partagées dans mon ouvrage de 2006 sur les prédiscours. Je conclus ce rappel en soulignant que la théorie de l'interpellation idéologique est véritablement une origine, une origine théorique pour la linguistique du discours, mais également pour la théorie du genre élaborée dans le terreau des cultural studies américaines. 


\section{3. "To call someone a name » : la construction sociale du genre}

To call someone a name veut dire à la fois « donner un nom » et « insulter », et c'est une expression qui permet à Judith Butler, on va le voir, de mettre en place sa théorie de la performativité.

\subsection{French theory : le retour au bercail}

On sait que vers le milieu des années 1980, la France, comme l'explique Erwann Sommerer (2005), abandonne l'analyse des idéologies avec une grande part du projet structuraliste. Il y a, sur le plan théorique, un blanc français, dont on se rend bien compte quand on fait l'histoire de l'analyse du discours: à partir de cette période, les choses changent radicalement, parce que Michel Pêcheux disparaît, et avec lui le groupe de discursivistes qui s'éparpille dans d'autres travaux et d'autres disciplines, mais aussi parce que Mikhail Bakhtine est projeté sur la scène du discours sans toujours ménager la chèvre de l'inconscient et le chou de la langue. C'est Jacqueline Authier qui souligne ainsi avec sévérité dans sa thèse de 1995 ces deux manques de Bakhtine, manques bientôt recouverts par le succès de la notion de dialogisme, promue et exploitée en profondeur par la linguistique textuelle mais surtout par l'interactionnisme.

Que deviennent alors la théorie de l'idéologie, le concept d'interpellation, celui d'assujettissement, en dehors des cercles marxistes confidentiels qui continuent de travailler dans une certaine obscurité ? Ils font l'objet, par delà l'Atlantique, de ce que Judith Butler appelle une «drôle de construction américaine » dans la seconde préface qu'elle donne en 1999 à Gender trouble (daté de 1990 et toujours non traduit en français à l'époque). Aux États-Unis se développe en effet un "post-marxisme ", selon l'expression de Erwan Sommerer, présenté dans le monde anglo-saxon comme une forme de "poststructuralisme », ce dernier, comme on sait, n'ayant jamais existé qu'aux États-Unis, avant même que le structuralisme n'ait presque eu le temps de s'y installer. Dans un article de 1992, un des rares qui porte sur les apports explicites de Michel Pêcheux aux cultural studies, Martin Montgomery et Stuart Allan (1992) expliquent comment le travail de Pêcheux, parce qu'il s'appuie sur la pensée d'Althusser en lui donnant une traduction discursive, a pu informer des travaux sur les formes discursives du pouvoir.

\subsection{De l'interpellation à la performativité du genre}

La gender theory mise en place par Judith Butler, professeure de rhétorique et de littérature à Berkeley en Californie, est issue de la théorie des idéologies et particulièrement des concept d'interpellation et d'assujettissement plutôt via Foucault (Le Blanc 2004) : l'assujettissement y est traduit en assignation de genre, y compris dans ses dimensions linguistiques, discursives et lexicales. Mais cette traduction est le résultat d'un travail de reconceptualisation élaboré, cette fameuse « drôle de construction » qui reformule l'interpellation en performativité.

La théorie de la performativité est présentée par Butler d'abord dans Gender trouble, puis connaît de nombreux approfondissements dans ses ouvrages postérieurs. Elle constitue bien sûr un retravail de la notion que J.L. Austin propose en 1962 How to do things with 
words. Elle y reprend centralement la notion d'effet, pour qualifier le genre et montrer qu'il est, de manière essentielle, une construction idéologique :

La prémisse de base ici, c'est que l' "être » du genre est un effet, un objet d'enquête généalogique qui vise à esquisser les paramètres politiques de sa construction sur le mode de l'ontologie. Dire que le genre est construit ne revient pas à dire qu'il est une illusion ou un pur artifice; ce serait placer ces termes à l'intérieur d'un couple de contraires dans lequel « réel » serait le contraire d' " authentique ». En tant que généalogie de l'ontologie du genre, cette enquête cherche à comprendre la production discursive du caractère plausible de ce rapport binaire; elle nous fait comprendre que certaines configurations du genre prennent la place du "réel », qu'elles consolident et étendent leur hégémonie lorsque ce processus performatif parvient à se naturaliser lui-même de manière heureuse (Butler 2005 [1990] : 109).

Comme les artisans français de la théorie du discours des années 1960 et 1970, elle donne la primauté au discours, et définit le genre comme identité sociale :

51 Dire que le corps genré [gendered] est performatif veut dire qu'il n'a pas de statut ontologique indépendamment des différents actes qui constituent sa réalité. Si cette réalité est constituée comme une essence intérieure, cela implique que cette intériorité est précisément l'un des effets d'un discours fondamentalement social et public, de la régulation publique du fantasme par la politique de la surface du corps, du contrôle des frontières du genre entre intérieur et extérieur ; c'est ainsi que cette intériorité institue l' « intégrité » du sujet (Butler 2005 [1990] : 259).

52 La meilleure définition de la performativité du genre se trouve dans le Pouvoir des mots et c'est un moment où Althusser est directement cité, Butler mentionnant la « scène de l'interpellation » althussérienne :

[...] l'existence sociale du corps est d'abord rendue possible par son interpellation à l'intérieur des termes du langage. Pour le comprendre, il nous faut imaginer une scène impossible, celle d'un corps qui n'a pas encore été socialement défini, un corps auquel, à rigoureusement parler, nous n'avons pas accès, et qui néanmoins devient accessible à l'occasion d'une adresse, d'un appel, d'une interpellation qui ne le « découvre » pas, mais qui, fondamentalement, le constitue (Butler 2004 [1997]: 26).

Dans un article de 2004 sur l'assujettissement où il met en rapport Althusser, Foucault et la postérité que leur donne Judith Butler, Guillaume Le Blanc précise que, dans l'interpellation, ce qu'il y a de plus fascinant est que le sujet se retourne, à tous les coups, ce qui donne à l'interpellation toute sa matérialité. L'adhésion à l'assujettissement est un phénomène que la french theory française n'a guère traité et pour laquelle la french theory américaine propose une interprétation: pour Judith Butler, dans La vie psychique du pouvoir, qui est une réinterrogation de la psychanalyse dans sa capacité à permettre une analyse du pouvoir, c'est la notion d'attachement, au sens psychanalytique du terme, c'est-à-dire l'attachement aux objets primordiaux que sont les parents. Je trouve l'hypothèse un peu faible, par rapport à l'élaboration robuste de ses autres propositions, en particulier en ce qui concerne la « resignification ». Pour elle en effet, l'interpellation agressive, comme l'insulte par exemple, n'est pas entièrement négative dans la mesure où elle permet au sujet de se constituer en répondant. Cette réponse est une inscription dans le langage, une manière d'être au langage qui assure la subjectivité. Un des exemples qu'elle développe dans Le pouvoir des mots est l'inversion des valeurs des interpellatifs à l'œuvre dans le processus de «resignification» par lequel les minorités humiliées se 
construisent des noms identitaires « récupérés » des insulteurs : c'est le cas du célèbre négritude, mais aussi de queer, ancienne insulte devenue étendard identitaire ou, en France, dans le domaine féministe, de chiennes (de garde) ou de salopes (dans l'expression « 343 salopes » de Charlie-hebdo pour désigner les 343 femmes qui signent le manifeste de 1971 en faveur de l'avortement). Judith Butler développe par ailleurs et sur un autre thème, dans son dernier ouvrage Le récit de soi, une perspective analogue. Pour résumer rapidement son propos, elle suggère de considérer l'activité de se raconter comme une activité essentiellement articulée sur l'autre, un tu, une forme d'interpellation par laquelle le sujet se constitue. On est loin de la conception française plus classique de l'écriture de soi. Cette conception du sujet, que Judith Butler ne relie pas à une perspective cognitive, est cependant cohérente avec une perspective qui inscrit l'interpellation dans un environnement cognitif.

\subsection{Le sujet en deuxième personne : moi-même comme toi autre}

En effet, l'appel à l'inconscient me semble nécessaire pour justifier l'adhésion idéologique, mais l'appel au contexte, ce que j'appelle dans une perspective cognitive, environnement cognitif, me semble plus pertinent: si en effet l'interpellation est une intersubjectivité, cette intersubjectivité constitue un environnement cognitif favorable pour un certain nombre de phénomènes liés à l'interaction, et défavorable pour d'autres.

Dans une perspective cognitive (dans le cadre de la cognition sociale et non de la cognition internaliste orthodoxe), l'interaction est vue comme un élément synthétique et non analytique. Ce que je veux dire, c'est qu'une perspective énonciativocommunicationnelle et/ou pragmatique sur l'interpellation, qui rend saillants le locuteur et le destinataire, en insistant sur la dimension illocutoire par exemple, ou sur la morphologie des éléments langagiers, présente l'interpellation de manière assez traditionnelle comme un procès clivé entre l'émission et la réception, même sous les catégories de la co-énonciation ou de la co-construction qui maintiennent le clivage entre les protagonistes. Dans une perspective cognitivo-discursive issue de la cognition sociale, il existe un continuum entre les deux protagonistes, dont l'échange et la coprésence forme un environnement cognitif, dans lequel j'intègre aussi le corps, pris en compte comme une donnée de l'échange et non seulement comme un contexte ou un accompagnement de la communication, ainsi que l'ensemble de l'environnement non humain. Cela veut dire que l'interpellation est de l'ordre de la cosubjectivité, et que ses conditions de félicité (c'est-à-dire le fait le fait que l'individu se retourne) ne dépendent ni seulement des contenus et formes langagiers, ni des statuts des protagonistes, ni de la reconnaissance de l'intention du locuteur, mais de la configuration d'un environnement global. Dans une perspective externaliste (par exemple Clark and Chalmers 1998) qui place l'esprit humain en dehors de lui-même et en particulier dans l'esprit de l'autre, le sujet est pensé comme collaboratif et non plus individuel, il est directement articulé sur l'environnement, qui intègre l'esprit de l'autre comme un de ses éléments. Cette position est bien développée désormais et représentée par exemple par les travaux de Bruno Latour ou de François Flahaut, le paradoxe de Robinson. Mais, sur ce point, les externalistes défendent, me semble-t-il, une conception assez ancienne, que l'on trouve éparpillée ça et là, chez Peirce (1930) ou chez Lacan par exemple : « Le moi est référentiel à l'autre. Le moi se constitue par rapport à l'autre. Il en est corrélatif », déclare-t-il dans 
le Séminaire 1 p. 83 ; ou encore Francis Jacques, dans une tradition encore différente, qui est la filiation lévinassienne :

Un homme qui se soustrait à l'interpellation ou qui interpelle autrui sans lui donner la parole à son tour, sans placer son propos sur le trajet des réponses possibles [....] n'actualise pas sa compétence pragmatique. En toute rigueur, il ne tient pas un comportement linguistique mais un comportement pré-linguistique de violence, à peine un comportement. Est violente toute action - verbale aussi bien - où j'agis comme si j'étais seul à agir, comme si le reste de l'univers n'était là que pour recevoir l'impact de mon action. Est ressenti dans la peur tout acte - de langage aussi bien - que je subis sans en être collaborateur (Jacques 1979 : 175).

\section{Conclusion}

L'interpellation est véritablement une notion-origine dont la puissance théorique se manifeste de deux manières : sa capacité à engendrer des notions héritières ou dérivées comme le préconstruit, la reconnaissance ou la performativité du genre; sa capacité à permettre l'émergence d'une théorie autonome, dotée d'une vraie puissance explicative en même temps que d'une dimension politique, la gender theory.

Dans le cadre d'une philosophie du discours, elle constitue une notion centrale pour rendre compte de l'intersubjectivité et de la dimension éthique des échanges verbaux. Elle indique également que le discours, loin d'être un simple outil ou même une coconstruction entre les partenaires de l'échange, est un dispositif beaucoup plus large et complexe qui engage l'ensemble des facettes de la dimension humaine.

\section{BIBLIOGRAPHIE}

Achard-Bayle, G., Lecolle, M. 2008 (à par.). Sentiment linguistique et discours spontanés sur le lexique. Recherches linguistiques 30 : Université de Metz.

Achard-Bayle, G., Paveau, M.-A. (dir.). (2008 à par.). Linguistique populaire ?. Pratiques 139-140. Metz : CRESEF.

Althusser, L. (1970). « Idéologie et appareils idéologiques d'État, notes pour une recherche ». La pensée. Revue du rationalisme moderne 151, juin 1970. Paris : 3-38 (écrit en 1969).

Althusser, L. (1993). Écrits sur la psychanalyse. Paris : Stock/IMEC (1966 : « Trois notes sur la théorie des discours ", 111-171).

Butler, J. (2002 [1997]). La vie psychique du pouvoir. Paris : Léo Scheer.

Butler, J. (2004 [1997]). Le pouvoir des mots. Politique du performatif. Paris : Éditions Amsterdam. Butler, J. (2005 [1990]). Trouble dans le genre (Gender Trouble), trad. C. Kraus. Paris : La Découverte. Burke, P. (1998). « Langage de la pureté et pureté du langage ». Terrain 31, Un corps pur, en ligne sur http://terrain.revues.org/document3142.html.

Burke, P. (1999). « Les langages de la politesse ». Terrain 33, Authentique ?, en ligne sur http:// terrain.revues.org 
Burke, J., Porter R. (199). Language, self, and society : a social history of language. Polity Press. Clark, A., Chalmers, D. (1998). « The extended mind ». Analysis 58 (1) : 10-23.

Fuchs, C., Pêcheux M. s.d. (1971 ?). La détermination : relatives et déterminants, mémoire s.l.n.d., $46 \mathrm{p}$.

Henry, P. (1975). « Constructions relatives et articulations discursive ». Langages 37, « Analyse du discours. Langue et idéologie » : 81-98.

Jacques, F. (1979). Dialogiques, Recherches logiques sur le dialogue. Paris : PUF.

Lacan, J. (1975 [1953-1954]), Séminaire 1, Les écrits techniques de Freud. Paris : Éditions du Seuil.

Lacan, J. (1978 [1954-1955]). Séminaire 2, Le moi dans la théorie de Freud et dans la technique de la psychanalyse. Paris : Éditions du Seuil.

Le Blanc, G. (2004). « Être assujetti : Althusser, Foucault, Butler ». Actuel Marx, 2/36 : 45-62.

Maldidier, D. (prés.) (1990). L’inquiétude du discours. Textes de M. Pêcheux. Paris : Éditions des Cendres.

Mazière, F. (2005). L'analyse du discours. Histoire et pratique. Paris : PUF, “Que sais-je ?".

Moïse, C. et al. (dir). (2008). La violence verbale, tome $1:$ "Espaces politiques et médiatiques », tome 2 : « Des perspectives historiques aux expériences éducatives », Paris : L'Harmattan, coll. « Espaces discursifs ».

Montandon, A. (dir.) (1995). Dictionnaire raisonné de la politesse et du savoir-vivre du Moyen Âge à nos jours. Paris : Seuil.

Montgomery, M., Allan S. (199). « Ideology, Discourse, and Cultural Studies : The Contribution of Michel Pêcheux ». Canadian Journal of Communication, 17/2.

Paveau, M.-A. (2006). Les prédiscours. Sens, mémoire, cognition. Paris : Presses Sorbonne nouvelle.

Paveau, M.-A. (2007), « Discours et cognition. Les prédiscours entre cadres internes et environnement extérieur », numéro spécial de la revue électronique Corela (Cognition, Représentation, langage) : «Contextes, discours, cognitions », codirigé avec G. Achard-Bayle, http://revue-corela.org

Paveau, M.-A. (2008). « Le parler des classes dominantes, objet linguistiquement incorrect? Dialectologie perceptive et linguistique populaire ", Études de linguistique appliquée : 138-156.

Paveau, M.-A., Rosier, L. (2008). La langue française. Passions et polémiques. Paris : Vuibert.

Pêcheux, M. (197). Les vérités de La Palice. Paris : François Maspero, coll. « Théorie ».

Sommerer, E. (2005). «L'Ecole d'Essex et la théorie politique du discours : une lecture "postmarxiste" de Foucault », Raisons politiques, 3/19:193-209.

\section{RÉSUMÉS}

Dans la perspective d'une philosophie du discours, qui implique nécessairement une histoire et même une éthique du discours, cet article se propose d'inscrire l'interpellation dans le cadre de l'intersubjectivité et de rendre compte de son fonctionnement, comme forme intersubjective nécessaire, dans trois domaines disciplinaires privilégiés, qui sont des lieux d'articulation entre la linguistique et ses disciplines affines ou contributives. Il s'agit de : l'histoire sociale du langage 
(en fait la Social history of language britannique, champ qui n'existe pas véritablement en France comme tel mais qui correspondrait à quelque chose comme une sociolinguistique ou une sociopragmatique historique), la théorie du discours marxo-freudienne (projet qui articule la linguistique, la philosophie et la psychanalyse entre 1960 et 1985) et, de l'autre côté de l'Atlantique, la gender theory, au croisement de la philosophie, de la rhétorique et de la pragmatique, qui développe une théorie de la performativité.

This paper proposes a discourse philosophy approach of interpellation, which engages an history of discourse, and even an ethic reflexion about the use of language. Interpellation is considered as a necessary form of intersubjectivity, which functioning needs the contribution of three disciplinary domains to be wholly described. These three domains are close to linguistics to which they bring a social and philosophical dimension in order to extend its explanatory power : the Social history of language, a british current which does not exist in France, but would perhaps corresponds to something as an historical sociolinguistics or pragmatics; the Freudomarxian theory of discourse (whichs links linguistics, philosophy and psychoanalysis in the structuralist period between 1960 and 1985 in France); and at last the American gender theory, where philosophy, rhetorics and pragmatics are crossing in the nineties to redefine in a poststructural way through the "french theory" some of the main concepts of american pragmatics as the Austinian performativity.

INDEX

Mots-clés : inconscient, norme sociale, philosophie du discours, performativité

Keywords : unconscious, social norm, discourse philosophy, performativity

\section{AUTEUR}

\section{MARIE-ANNE PAVEAU}

Université de Paris 13 EA 459 Cenel 